\title{
Reanalysis of Voyager 2 UVS Occultations at Uranus: Hydrocarbon Mixing Ratios in the Equatorial Stratosphere
}

\author{
J. BISHOP AND S. K. ATREYA \\ Department of Atmospheric, Oceanic and Space Sciences, University of Michigan, \\ Ann Arbor, Michigan 48109-2143

\section{F. HERBERT} \\ Lunar and Planetary Laboratory West, University of Arizona, Tucson, Arizona 85721
}

\author{
AND \\ P. ROMANI \\ Science Systems and Applications, Inc., Seabrook, Maryland 20706
}

Received December 5, 1989; revised July 2, 1990

\begin{abstract}
Application of noise filtering and inversion techniques to single-channel UVS lightcurves obtained during the Voyager 2 solar occultation at Uranus has yielded tighter constraints on the structure and composition of the upper equatorial stratosphere at the time of the encounter. Specifically, atmospheric pressure and temperature profiles in the altitude region bracketted by total number densities $2 \times 10^{15} \mathrm{~cm}^{-3}$ and $5 \times 10^{16} \mathrm{~cm}^{-3}$ have been derived, based on the observed $\mathrm{H}_{2}$ Rayleigh scattering opacity profiles (wavelengths $>$ $153 \mathrm{~nm}$ ) with an assumed helium mixing ratio of 0.15 : at the density level $3.3( \pm 0.2) \times$ $10^{16} \mathrm{~cm}^{-3}$, the pressure is $0.60( \pm 0.01)$ mbar with a temperature $133( \pm 8) \mathrm{K}$. Lightcurves obtained at shorter wavelengths reveal the mixing ratio of acetylene to be increasing $(0.7$ $\times 10^{-8}-1.5 \times 10^{-8}$ ) with increasing pressure in the pressure interval $0.10-0.30$ mbar. Ethane is also present at these pressures with a mixing ratio of roughly $10^{-8}$; if methane is present at mixing ratios in excess of roughly $3 \times 10^{-7}$, then the ethane estimate may need to be halved. Comparison with photochemical models indicates values of $10^{3}-10^{4}$ $\mathrm{cm}^{2} \mathrm{sec}^{-1}$ for the eddy mixing coefficient at the methane homopause, depending on the manner in which eddy mixing is assumed to vary with atmospheric number density. It has not been possible to obtain meaningful results from the stellar occultations, which are characterized by poor signal-to-noise ratios. 1990 Academic Press, Inc.
\end{abstract}

\section{INTRODUCTION}

During the Voyager 2 encounter with Uranus in January 1986, one solar (ingress) and three stellar (ingress and egress for $\gamma$ Pegasus, grazing for $\nu$ Geminorum) occultations were recorded with the ultraviolet spectrometer (UVS) (Broadfoot et al. 1986). The solar and $\gamma$ Pegasus occultations have undergone prior analysis using modeling tech- niques (Herbert et al. 1987). Specifically, model atmospheres incorporating methane photochemistry were constructed and the extinction of the solar or stellar FUV spectrum by the model atmosphere was compared with the observed transmission as the spacecraft passed behind the planet. It was not only possible to ascertain the density profile of $\mathrm{H}_{2}$ and exobase temperature high in the atmosphere, as had been done with 
occultation data at Jupiter and Saturn (Broadfoot et al. 1981, Atreya et al. 1981, Festou and Atreya 1982) but also to extend the determination of temperature and $\mathrm{H}_{2}$ density down to stratospheric levels. This ability is a direct consequence of the relative paucity of hydrocarbons in the Uranian upper atmosphere. In fact, $\mathrm{H}_{2}$ Rayleigh scattering (with a cross section $2.7 \times 10^{-25} \mathrm{~cm}^{2}$ near $160 \mathrm{~nm}$ ) is the only opacity source evident in the UVS occulation lightcurves at wavelengths longer than $155 \mathrm{~nm}$. Lightcurves at shorter wavelengths give clear evidence for the presence of expected hydrocarbon species (primarily acetylene, ethane, and methane). However, due to overlap in absorption spectra and a lack of altitude resolution in the lightcurves used for comparison with models, it was not possible in Herbert et al. (1987) to say much more than that hydrocarbons were present and to give "representative"' values of equatorial $\mathrm{C}_{2} \mathrm{H}_{2}$ and $\mathrm{C}_{2} \mathrm{H}_{6}$ mixing ratios $\left(10^{-8}\right.$ and several $\times 10^{-8}$, respectively) for the altitude range $200-400 \mathrm{~km}$ above the 1.0-bar level.

In view of the significance of stratospheric hydrocarbons as tracers of the strength of eddy mixing and in haze production (Pollack et al. 1987, Atreya et al. 1990), the UVS solar occultation lightcurves at wavelengths longer than $135 \mathrm{~nm}$ have been reanalyzed to extract hydrocarbon abundances. The analysis scheme employed differs from that used in Herbert et al. (1987) and entails the application of a standard time series filtering technique to estimate and remove the noise component in individual lightcurves. In this way, it is possible to extract sequentially $\mathrm{H}_{2}$ and hydrocarbon column densities along the line of sight. Number density profiles have been obtained using an Abel inversion procedure. The resulting hydrocarbon mixing ratios are then compared with photochemical models to infer the strength of eddy mixing, using an empirical stratosphere model. This procedure works well for the solar occultation data set, which has a relatively high signal-to-noise ratio. The stellar occultation data are of much poorer quality in this regard, and it has not been possible to obtain meaningful results for these data sets.

\section{DATA HANDLING}

A complete description of the UVS instrument is contained in Broadfoot et al. (1977) and Broadfoot et al. (1981); only those aspects directly bearing on our analysis will be noted here. In occultation mode, the UVS instrument registers a complete spectrum spanning $53-170 \mathrm{~nm}$ at $0.32-\mathrm{sec}$ intervals, with a nominal spectral resolution of 0.93 $\mathrm{nm}$. The realized spectral resolution is 2.5 $\mathrm{nm}$ for a point source. Spatial (height) resolution is determined in general by the sampling rate and the drift speed of the minimum tangent ray height (MTRH, defined as the altitude of closest approach of the line of sight to planet center). For the $\gamma$ Pegasus ingress occultation, the drift speed was 17.8 $\mathrm{km} \mathrm{sec}^{-1}$, giving a nominal height resolution of $5.6 \mathrm{~km}$. The solar occultation drift speed ranged from 8.2 to $7.6 \mathrm{~km} \mathrm{sec}^{-1}$ for a nominal resolution of $\sim 2.5 \mathrm{~km}$; however, the Sun is not a point source and subtended an apparent width of $82 \mathrm{~km}$ within the atmosphere as seen by the UVS at the time of occultation. The realized height resolution in the solar occultation is estimated to be better than $10 \mathrm{~km}$, using the solar disk deconvolution procedure described below. Throughout, we measure altitudes in reference to the 1.0-bar level, placed at an equatorial planetocentric radius of $25,559 \mathrm{~km}$ by the radio science subsystem (RSS) measurements (Lindal et al. 1987).

Examples of lightcurves obtained by the UVS instrument during the solar occultation are shown in Fig. 1. These represent the progressive extinction of light received by the respective channels as greater columns of gas intervene along the line of sight, and directly give the atmospheric opacity as a function of MTRH. In general, several molecular species will contribute to the opacity at any given wavelength; by comparing opacities at several wavelengths for constant MTRH, the constituent opacities and column densities can be unraveled, once in- 


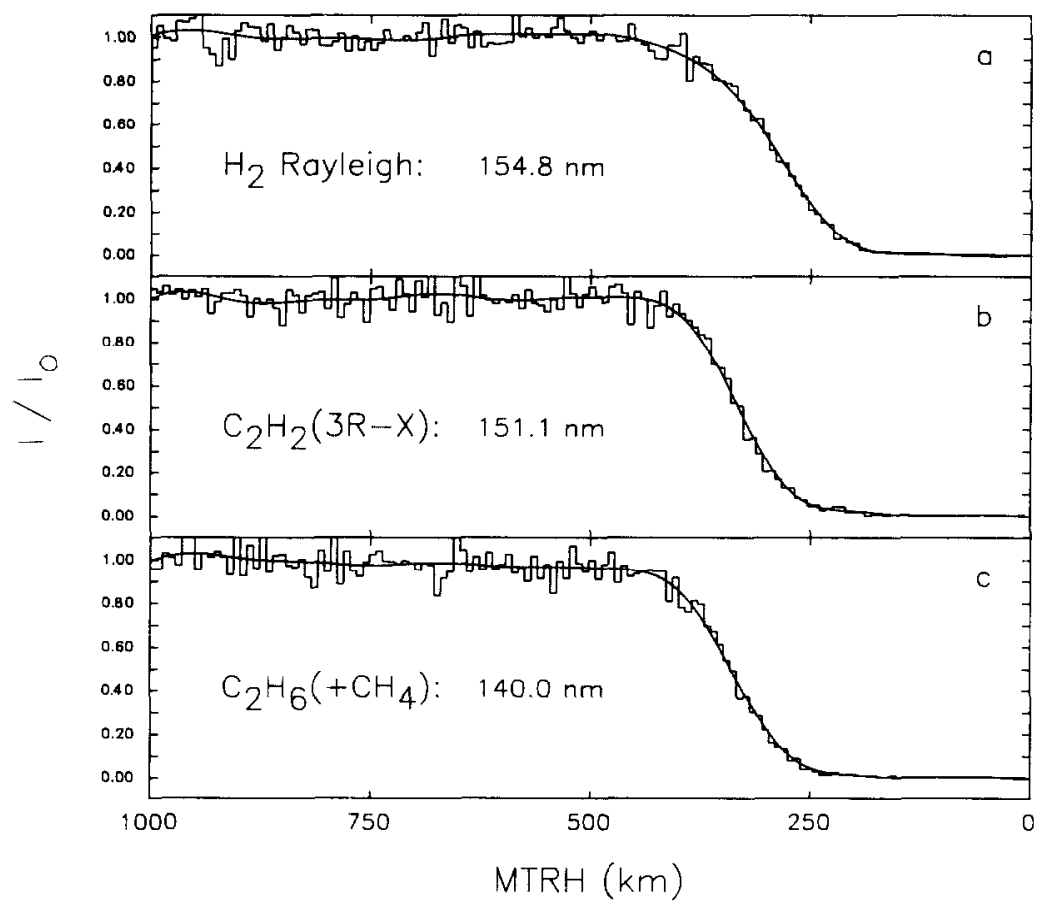

FIG. 1. Solar occultation lightcurves for three channels, identified by center wavelength with channel offset applied (see text). Binning over three raw spectra has been applied, for a nominal altitude resolution of $7.3 \mathrm{~km}$. The filtered, deconvolved counterparts used in the analysis are also shown. (a) Extinction entirely attributable to Rayleigh scattering by $\mathrm{H}_{2}$. (b) Lightcurve for the channel with the first vibrational member of the $3 R-X$ Rydberg transition of acetylene nearest to channel center. (c) Lightcurve exhibiting additional opacity attributable to ethane and possibly methane. The lightcurves fed into the filtering procedure comprised 256 sequential $I / I_{0}$ measurements centered on the half-light point. Abscissa is altitude in kilometers above the 1.0-bar level.

strumental effects have been taken into account. Such effects (primarily noise and $I_{0}$ fluctuations) are evident in the data at MTRHs above the onset of extinction.

The data output from each channel constitutes a simple time series, suggesting the use of standard analysis techniques to estimate and filter out the respective noise component. The technique we have chosen involves constructing an "optimal filter" for each channel lightcurve, as described in Press et al. (1986, Chapter 12); in particular, the power spectral density (PSD) has been estimated using the MEM technique. In Fig. 2, PSDs obtained in this way for a channel dominated by $\mathrm{H}_{2}$ Rayleigh scattering are compared with the PSD calculated using a representative $\mathrm{H}_{2}-\mathrm{He}$ model atmosphere (Herbert et al. 1987, Table 1, solar occultation case). The point is that the expected form for the "true" PSD is a rapidly decreasing function of frequency, with noise dominating the channel PSDs at frequencies above $\sim 0.05 \mathrm{~Hz}$, so that the appropriate optimal filter is readily constructed. Also, by working with fourier transforms of each lightcurve, the solar disk deconvolution is easily effected by segmenting the disk into bands of width $7.3 \mathrm{~km}$ (the nominal altitude resolution after height compression). In this, we have assumed the solar disk to be uniformly bright, which seems reasonable for the solar minimum epoch of the encounter, and that it remained stationary in the 


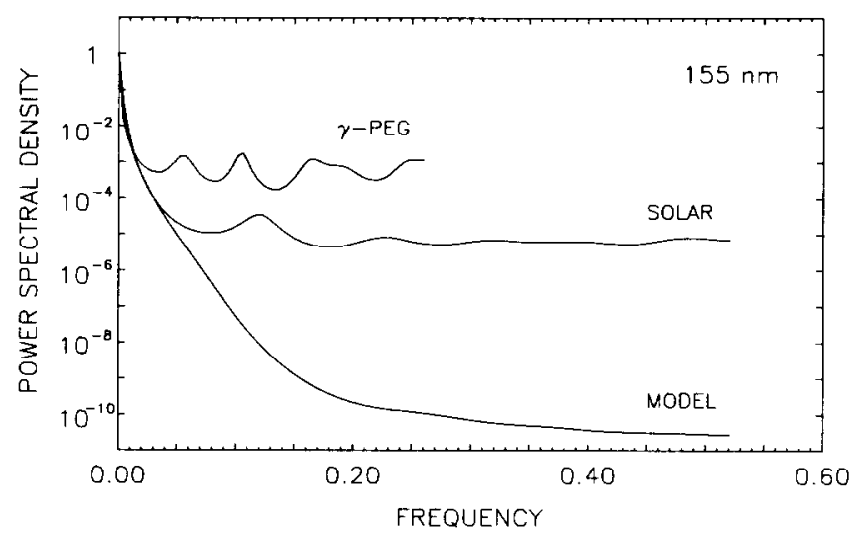

FIg. 2. Representative power spectral densities (PSDs) for the solar and $\gamma$ Pegasus ingress occultations, for the respective channel centered near $155 \mathrm{~nm}$. These have been normalized to the DC (zero frequency) value and are compared with the PSD belonging to the $155-\mathrm{nm}$ lightcurve given by the stratosphere model of Herbert et al. (1987, Table 1, solar occultation case). This is to illustrate the frequency limits and noise levels that must be recognized when constructing "optimal" filters. Six $\gamma$ Pegasus occultation spectra were compressed to obtain the PSD shown, for a nominal altitude resolution of $34 \mathrm{~km}$.

entrance aperture. Trial deconvolutions were also performed for solar disks with limb darkening; this exercise resulted in variations in retrieved densities that are minor compared with other sources of uncertainty discussed below.

Prior to noise filtering, the UVS data sets were compressed by binning over a small number of raw spectra. The solar occultation lightcurves used in our analysis are averages over three raw spectra for an effective sampling interval of $0.96 \mathrm{sec}$, imposing a Nyquist fourier resolution limit of $\sim 15 \mathrm{~km}$. As illustrated in Fig. 2, the signal contained in the solar occultation lightcurves is confined to fourier components of much longer spatial extent, so the filtering procedure is not compromised. In this case, height compression is carried out mainly to reduce the size of the data set, and the resultant altitude resolution is determined primarily by the MTRH drift speed and secondarily by the solar disk deconvolution procedure. The poorer signal-to-noise ratio $(\mathrm{S} / \mathrm{N})$ of the stellar occultations force a more drastic degree of compression. However, the respective drift speeds impose an upper limit to the number of spectra that can be binned if any information on altitude distributions is to be retained. For the ingress $\gamma$ Pegasus occultation, this limit is roughly 18 spectra since evidence of hydrocarbons will be confined to altitudes within roughly $100 \mathrm{~km}$ above the last light level for $\mathrm{H}_{2}$ Rayleigh scattering. As discussed below, even this degree of altitude compression, augmented by channel summation, failed to yield a composite $\mathrm{S} / \mathrm{N}$ high enough to obtain meaningful results with our filtering approach; the other stellar occultations are of worse quality.

Lastly, due to residual pointing uncertainties and inaccurate removal of low-frequency noise in the filtering process, it is not possible to establish the true $I_{\mathrm{o}}$ level. To compensate, the lightcurves shown in Fig. 1 are scaled so that the mean value of $I / I_{\mathrm{o}}$ in the altitude range $1000-700 \mathrm{~km}$ (atmospheric pressures $<1.0 \mu$ bar) is unity.

\section{ANALYSIS}

\section{Extraction of Column Densities}

Prior work has established that hydrocarbon densities throughout the pressure range probed during the solar occultation remain 
too small to affect the extinction at wavelengths longward of $153 \mathrm{~nm}$; these lightcurves thus provide the line of sight (LOS) $\mathrm{H}_{2}$ column density directly. In our analysis, the channels spanning 155-162 nm (Fig. 1a) have been separately filtered and the corresponding $\mathrm{H}_{2}$ column densities $\hat{N}_{\text {LOS }}\left(\mathrm{H}_{2}\right)$ obtained using the relation

$$
\mathcal{N}_{\text {LOS }}(z)=-\frac{1}{\sigma} \ln \left(\frac{I(z)}{I_{\mathrm{o}}}\right)_{\mathrm{f}},
$$

where $z$ is the minimum tangent ray height, $\sigma$ is the Rayleigh scattering cross section evaluated at the channel center wavelength, and the subscript $f$ denotes the filtered lightcurve. Throughout, we use the theoretical Rayleigh scattering cross sections of Ford and Browne (1973) to relate $\mathrm{H}_{2}$ column densities to $\mathrm{H}_{2}$ Rayleigh opacity; note that Rayleigh scattering cross sections at wavelengths longward of $100 \mathrm{~nm}$ can be treated as constant over $2.5 \mathrm{~nm}$ wavelength increments (i.e., the width of the UVS slit function) with errors $<10 \%$. The individual $\mathcal{N}_{\text {LOS }}\left(\mathrm{H}_{2}\right)$ profiles were then averaged together. Results are shown in Fig. 3 for MTRHs satisfying $0.10<\tau_{\text {LOS }}<2.00$ where $\tau_{\mathrm{LOS}}$ is the total optical depth along the line of sight.

The various $\mathcal{N}_{\text {LOS }}\left(\mathrm{H}_{2}\right)$ profiles presented in Fig. 3 illustrate the sensitivity of the procedure to filter parameters. Profiles " $A$ " are the main results; profiles " $B$ " and " $C$ " are shown to indicate the sorts of profiles generated by ill-suited filters. The filters used in obtaining the profiles of group "A" (three in number) differ in the low-frequency noise estimate; the extrapolation of the noise amplitude to zero frequency is the most significant source of uncertainty in the filtering procedure. The lack of differentiation among these profiles argues well for this application of noise filtering; filter parameters were based on accurate PSD estimates, and enough fourier components were retained in the filtering procedure to permit a believable reconstruction of the underlying lightcurve.
Profile " $B$ " results when a filter removes too many fourier components (in this example, by filtering as if the relative noise level were as great as in the $\gamma$ Pegasus ingress occultation (Fig. 2)), while profile " $\mathrm{C}$ " is an example with too many fourier components retained resulting in the appearance of relatively small-scale, spurious perturbations (i.e., the noise spectrum is altered but not entirely removed).

The conversion of $I / I_{\mathrm{o}}$ to column densities $\mathcal{N}_{\text {LOS }}(z)$ is more complicated when the molecular photoabsorption spectrum exhibits discrete lines or bands $\left(\mathrm{C}_{2} \mathrm{H}_{2}\right)$ or varies rapidly with wavelength $\left(\mathrm{C}_{2} \mathrm{H}_{6}, \mathrm{CH}_{4}\right)$. It is necessary in these cases to model channel response with an interpolation table of extinction vs column density, done by convolving the molecular photoabsorption spectrum with an assumed incident solar or stellar irradiance spectrum and the UVS slit function in an average over each channel wavelength interval. Here we have used a reference solar minimum irradiance spectrum given by Heroux and Hinteregger (1978), and the hydrocarbon photoabsorption cross sections are those used in the analysis of UVS data from previous planetary encounters (see Fig. 2 of Herbert et al. (1987); also Fig. 2.16 of Atreya (1986)). A further complication in extracting equatorial $\mathrm{C}_{2} \mathrm{H}_{2}$ LOS column densities arises from the Sun not being exactly centered in the UVS field of view during occultation. This spatial offset resulted in a channel (i.e., wavelength) offset relative to preflight calibration and other occultation observations that is not precisely known. The relation between extinction and column density for a discrete spectrum is fairly sensitive to the channel location of the spectral features, so that an uncertainty in location can translate into a fairly large uncertainty in the inferred column density.

Acetylene exhibits relatively large photoabsorption cross sections in the interval 143-152 nm, particularly at $151.9,147.8$, and $143.9 \mathrm{~nm}$ (vibrational progression of the 3R-X Rydberg transition), while ethane and 


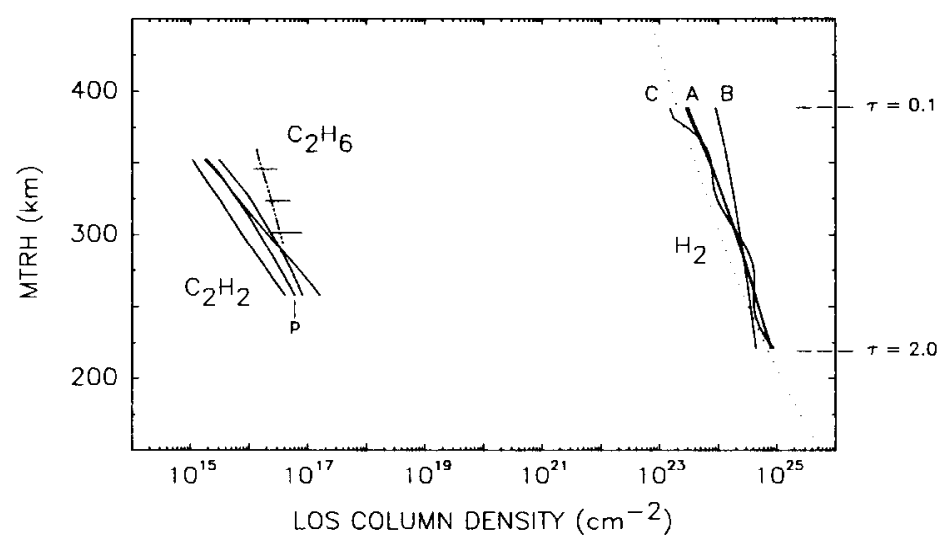

Fig. 3. Extracted column densities along the line of sight $\left(\mathcal{N}_{\mathrm{LOS}}\right)$ for $\mathbf{H}_{2}, \mathrm{C}_{2} \mathrm{H}_{2}$, and $\mathrm{C}_{2} \mathrm{H}_{6}\left(+\mathrm{CH}_{4}\right)$, obtained with filtered solar occultation lightcurves and plotted against minimum tangent ray height (MTRH, in kilometers above the 1-bar level). For $\mathcal{N}_{10 S}\left(\mathrm{H}_{2}\right)$, the profiles of group " $\mathrm{A}$ " are the main results; profiles " $B$ " and " $C$ " are shown to illustrate features associated with ill-suited filters. The $\mathcal{N}_{\text {LOS }}\left(\mathrm{C}_{2} \mathrm{H}_{2}\right)$ profiles are derived from the lightcurve shown in Fig. 1b, for differing values of a channel (wavelength) offset related to pointing uncertainties experienced during the solar occultation (see text); the profile labeled " $p$ " is preferred in that it best accounts for the $\mathrm{C}_{2} \mathrm{H}_{2}$ opacity evident in neighboring channels. $\mathcal{N}_{\text {Los }}\left(\mathrm{C}_{2} \mathrm{H}_{6}\right)$ extractions are not as sensitive to the channel offset value; the indicated uncertainties are determined by this and by variations among the lightcurves used to obtain this profile (wavelengths $137-142 \mathrm{~nm}$ ). The dotted $\mathcal{N}_{\mathrm{LOS}}\left(\mathrm{H}_{2}\right)$ profile is from Herbert et al. (1987, Table 1, solar occultation case).

methane cross sections reach comparable values $\left(>10^{-17} \mathrm{~cm}^{2}\right)$ only below about 140 $\mathrm{nm}$ and $135 \mathrm{~nm}$, respectively. The lightcurve containing the $151.9 \mathrm{~nm}$ peak (Fig. 1b) has been used to derive the distribution of acetylene after correction for $\mathrm{H}_{2}$ Rayleigh opacity, subject to the conditions that $\mathcal{N}_{\text {Los }}\left(\mathrm{C}_{2} \mathrm{H}_{2}\right)$ exceeds $10^{15} \mathrm{~cm}^{-2}$ and $\mathcal{N}_{\text {LOS }}\left(\mathrm{H}_{2}\right)$ remains less than $4 \times 10^{24} \mathrm{~cm}^{-2}$. Solar occultation results are shown in Fig. 3. Each $\mathcal{N}_{\text {LOS }}\left(\mathrm{C}_{2} \mathrm{H}_{2}\right)$ profile in this figure is an average over profiles obtained with a small number of well-prescribed filters but extracted using different values of the channel offset centered on an estimate derived from the placement of spectral features at shorter wavelengths (e.g., the solar Lyman- $\beta$ line); the $\mathrm{H}_{2}$ LOS column density used for correction is the average of profiles "A." The profile labeled " $p$ " is based on an offset value that best accounts for the residual $\mathrm{C}_{2} \mathrm{H}_{2}$ opacity evident in neighboring channels and consequently is preferred.

The photoabsorption spectrum of ethane rises rapidly in the wavelength region of interest here while remaining relatively featureless. Lightcurves spanning the interval 137-142 nm should reflect the presence of this species, provided $\mathcal{N}_{\text {LOS }}\left(\mathrm{C}_{2} \mathrm{H}_{6}\right)$ exceeds $\sim 10^{16} \mathrm{~cm}^{-2}$ and $\mathcal{N}_{\text {LOS }}\left(\mathrm{H}_{2}\right)$ remains less than $2.5 \times 10^{24} \mathrm{~cm}^{-2}$. Acetylene cross sections at these wavelengths are relatively weak (3-4 $\times 10^{-18} \mathrm{~cm}^{2}$ ), as are those of methane. The 137-142 nm lightcurves (Fig. 1c) were individually filtered and corrected for $\mathrm{H}_{2}$ and $\mathrm{C}_{2} \mathrm{H}_{2}$ opacities based on the longer wavelength results for a range of channel offset values. The corresponding $\mathcal{N}_{\text {LOS }}\left(\mathrm{C}_{2} \mathrm{H}_{6}\right)$ profiles were obtained from the residual opacities by referring to an interpolation table analogous to the one used in the acetylene analysis and then were averaged over wavelength. The uncertainties associated with the $\mathcal{N}_{\text {LOS }}\left(\mathrm{C}_{2} \mathrm{H}_{6}\right)$ profile in Fig. 3 (obtained with the offset value associated with the $\mathcal{N}_{\text {LOS }}\left(\mathrm{C}_{2} \mathrm{H}_{2}\right)$ profile labeled " $p$ ") are determined from the spread among individual channel results and the use of different 
offset values. Some $\mathrm{CH}_{4}$ absorption may be contributing to this result; this possibility is discussed below in conjunction with derived $\mathrm{C}_{2} \mathrm{H}_{6}$ mixing ratios.

The $\mathcal{N}_{\text {LOS }}\left(\mathrm{H}_{2}\right)$ profile " $\mathrm{B}$ ' in Fig. 3 illustrates that noise removal is in itself not enough to guarantee that the resulting lightcurve will be a good approximation to the underlying signal: if the noise level is too high, then too few fourier components are retained to permit an adequate reconstruction of the signal. This has been the case with the stellar occultation lightcurves. Our attempts at extracting information from the $\gamma$ Pegasus ingress occultation data (latitude $63.7^{\circ} \mathrm{S}$ ) included summing both over altitude in the manner described earlier and over neighboring channels (e.g., channels spanning 153-162 nm for $\mathcal{N}_{\mathrm{LOS}}\left(\mathrm{H}_{2}\right)$ ). These manipulations did little to lower the relative noise level illustrated in Fig. 2. The resulting filtered lightcurves were inherently unable to replicate basic features of the data, for example, low dark count levels below 250 $\mathrm{km}$ altitude (referenced to the 1-bar radius of $25,100 \mathrm{~km}$ from Lindal et al. (1987, Fig. $8)$ ). This is unfortunate because the latitude sampled by this occultation is within the area observed by the UVS while obtaining an FUV airglow spectrum of the subsolar region, analysis of which has suggested strong latitudinal variations in the abundance and distribution of hydrocarbons in the Uranian stratosphere (Yelle et al. 1989). A direct comparison will not be possible.

Finally, it has not been possible to extend our analysis to wavelengths shorter than 135 $\mathrm{nm}$ for two reasons. First, the steady level of ethane opacity and the resurgence in $\mathrm{C}_{2} \mathrm{H}_{2}$ photoabsorption cross sections with decreasing wavelength (e.g., the vibrational progressions of the $3 R^{\prime}-X$ and $3 R^{\prime \prime \prime}-X$ Rydberg series), along with the steady increase in $\mathrm{H}_{2}$ Rayleigh scattering cross sections, leave no room for a clear identification of methane opacity, given the uncertainties in the analysis. Second, internal instrument scattering of the intense solar Lyman- $\alpha$ line affects channels below $135 \mathrm{~nm}$ in a way that can be only roughly corrected; the net effect is to lead to probable overestimates of hydrocarbon opacity by artificially elevating the $I_{\mathrm{o}}$ level and by steepening the apparent lightcurve as Lyman- $\alpha$ is progressively absorbed.

\section{$\mathrm{H}_{2}$-He Model Stratosphere}

The $\mathcal{N}_{\text {LOS }}$ profiles shown in Fig. 3 have been converted to number density profiles using an Abel inversion relation:

$$
\begin{aligned}
& n_{i} \approx\left(\frac{2}{\pi^{2} r_{\mathrm{o}} \Delta}\right)^{1 / 2}\left[\mathcal{N}_{\text {LOS }_{i}}\right. \\
&-\quad \sum_{j=1} \mathcal{N}_{\text {LOS } i-j}\left(2 j^{1 / 2}-(j+1)^{1 / 2}\right. \\
&\left.\left.-(j-1)^{1 / 2}\right)\right],
\end{aligned}
$$

where the index $i$ refers to sequential values of the minimum tangent ray height $z_{i}$ (ingress convention), $\Delta$ is the spacing in MTRH, and $r_{\mathrm{o}}$ is the 1.0-bar planetocentric radius. Equation (2) is derived from the standard Abel inversion integral (neglecting refraction effects; see, for instance, Hays and Roble 1968)

$$
n\left(z_{i}\right) \approx \frac{-1}{\pi\left(2 r_{\mathrm{o}}\right)^{1 / 2}} \int_{z_{i}}^{\infty}\left(z-z_{i}\right)^{-1 / 2} \frac{d \mathfrak{N}_{\operatorname{LOS}}(z)}{d z} d z
$$

by partitioning the integral over intervals defined by the $z_{i}$-sequence and approximating $d \mathcal{N}_{\mathrm{LOS}} / d z \approx\left(\mathcal{N}_{\mathrm{LOS}, i}-\mathcal{N}_{\mathrm{LOS}, i-1}\right) / \Delta$ in the interval $z_{i} \leq z<z_{i-1}$, which is valid when $\Delta$ $\ll \mathcal{N}_{\text {LOS }}\left(d \mathcal{N}_{\text {LOS }} / d z\right)^{-1}$. Tests with isothermal models replicating the Uranian occultation geometry indicate that retention of six or more terms in Eq. (2) leads to density estimates accurate to better than $10 \%$ for temperatures between $100 \mathrm{~K}$ and $200 \mathrm{~K}$. A series of eight terms has been used to obtain the number densities reported in this paper, to take advantage of the accuracy of the $\mathcal{N}_{\text {LOS }}\left(\mathrm{H}_{2}\right)$ determination (estimated to be better than $5 \%$ ).

The total number density profiles $n_{\mathrm{TOT}}(z)$ obtained using the "A" profiles of Fig. 3 are 


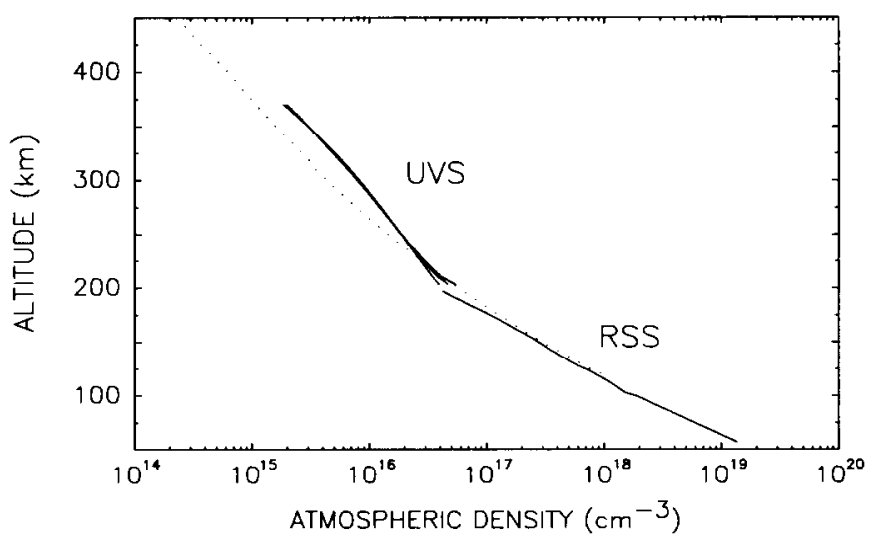

FIG. 4. Atmospheric number density profiles $n_{\text {TOT }}(z)$ obtained by inverting the $\mathcal{N}_{\text {LoS }}\left(\mathrm{H}_{2}\right)$ results " A" of Fig. 3, plotted against altitude above the 1.0-bar level (refer to Eq. (2)). Filter-related uncertainties are indicated by the variation among the individual profiles. The RSS number density profile is also shown, up to $200 \mathrm{~km}$ altitude (Lindal et al. 1987, Table 1). The dotted $n_{\text {TOт }}$ profile is from the solar occultation case model of Herbert et al. (1987).

shown in Fig. 4, assuming the mixing ratio of helium is 0.15 (Hanel et al. 1986). The variation among these profiles indicates the filter-related uncertainties involved. The RSS $n_{\mathrm{TOT}}(z)$ profile is shown for comparison, truncated near $200 \mathrm{~km}$ altitude (Lindal et al. 1987, Table 1). The UVS profiles span three scale heights of atmosphere $\left(2 \times 10^{15}\right.$ $\mathrm{cm}^{-3}<n_{\text {TOT }}<5 \times 10^{16} \mathrm{~cm}^{-3}$ ), so the pressure near the bottom of the observed range is reliably estimated using hydrostatic equilibrium: $0.60 \pm 0.01$ mbar with a temperature of $133( \pm 8) \mathrm{K}$ at the density level 3.3 $( \pm 0.2) \times 10^{16} \mathrm{~cm}^{-3}$. The uncertainties indicate the range of values obtained using different, equally plausible filters.

In the next section we discuss the results of photochemical models geared toward reproducing the observed hydrocarbon mixing ratios (described below). An empirical equatorial stratosphere model has been constructed for use in the photochemical calculations, based on the average of the UVS density profiles of Fig. 4 for altitudes above $200 \mathrm{~km}$ and the RSS results for densities greater than $2.0 \times 10^{17} \mathrm{~cm}^{-3}$ (altitudes below $160 \mathrm{~km}$ ). Inasmuch as density is the primary result of the occultation analyses, cubic spline interpolation between the UVS and RSS profiles has been used to prescribe density in the altitude interval $160-200 \mathrm{~km}$. Pressure-temperature profiles for this composite model are shown in Fig. 5. Temperature cannot be determined using a hydrostatic approach at altitudes near the $\tau_{\mathrm{LOS}}=$ 0.10 level, but the range of upper temperatures applied to the UVS number density profile encompass the values that might be expected. A crude estimate of $130 \mathrm{~K}$ for the upper temperature can be gleaned from the density lapse rate near $350 \mathrm{~km}$ altitude. This value has been used to finalize the model, the UVS and interpolated segments of which are given in Table $I$. The densities in this model are larger than the published RSS values at altitudes above $160 \mathrm{~km}$, with the differences remaining less than $10 \%$ at altitudes below $190 \mathrm{~km}$ and increasing to $\sim 25 \%$ at the base of the UVS range. This can be taken to be excellent agreement. The better characterization of the density profile above $200 \mathrm{~km}$ leads to a considerable increase in derived temperature at pressures less than 2.0 mbar compared with the published RSS model while continuing to exhibit a localized temperature maximum near 0.4 mbar.

The empirical model of Table I seems to be consistent with ground-based measure- 


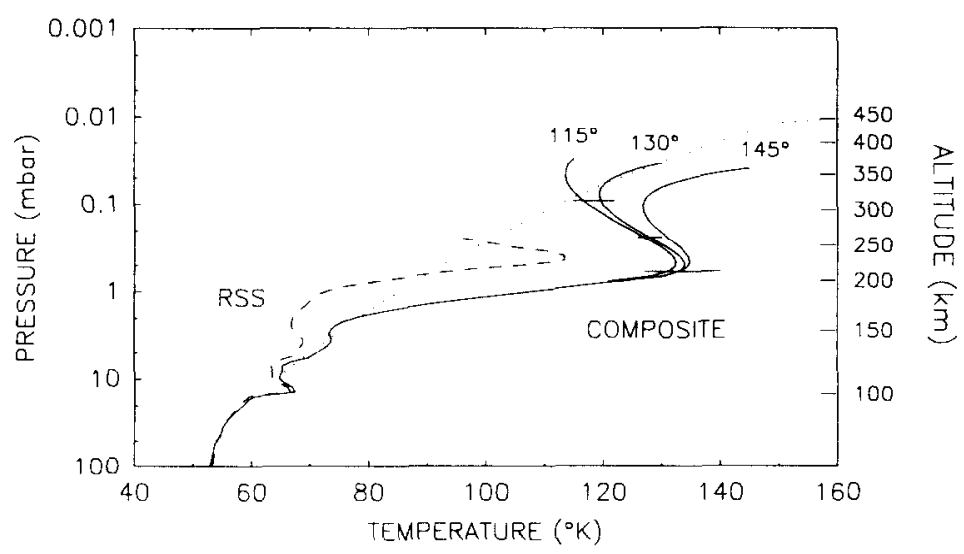

FIG. 5. Pressure-temperature profiles for the composite RSS and UVS stratosphere model, for a range of upper temperature values. The temperature uncertainties indicated for the UVS portion of the model belong to the profile with an upper temperature of $130 \mathrm{~K}$; these are based on variations among the temperature profiles derived by applying hydrostatic equilibrium (with the indicated upper temperature) to number density profiles obtained with different well-prescribed filters. The dashed curve is from the RSS model of Lindal et al. (1987, Table 1) and the dotted curve is from the solar occultation model of Herbert et al. (1987, Table 1), which has becn used to extend the composite model to lower pressures.

ments. For instance, the nominal case $p-T$ profile of Fig. 3 of Orton et al. 1987 (with a maximum temperature of $135 \mathrm{~K}$ and a temperature at 1.0 mbar of $109 \mathrm{~K}$ ) bears some resemblance to the composite profile of Fig. 5. Stellar occultation inversion results (French et al. 1983, Sicardy et al. 1985, Millis et al. 1987, French et al. 1987, Elliot et al. 1987) generally do not extend deeper than about 0.03 mbar, but the nominal upper temperature of $130 \mathrm{~K}$ in Fig. 5 is in line with these analyses. The warm layer near 0.008 mbar that has often been reported in stellar occultation analyses is above the pressure region probed by the UVS $\mathrm{H}_{2}$ Rayleigh lightcurves. However, reanalysis of $\mathrm{KME}$ 15 and KME 17 stellar occultation lightcurves using the UVS-derived thermosphere model of Herbert et al. (1987) to specify the atmosphere above the region probed by the stellar refraction lightcurves has lead to a considerable revision of the inferred temperature structure near and above 0.01 mbar (Baron and French 1989); the empirical model of Table I may be useful in further refinements of these analyses.

\section{Hydrocarbon Distributions}

Acetylene mixing ratios $f\left(\mathrm{C}_{2} \mathrm{H}_{2}\right)$ obtained from profile "p" of Fig. 3 using Eq. (2) in conjunction with the model stratosphere of Table I are shown in Fig. 6. The uncertainties indicated in this figure are based on variations exhibited when the channel offset value sweeps through its range of likely values, although it is our belief that the criterion leading to the selection of profile " $p$ " as preferred is fairly rigid, and on differences with $f\left(\mathrm{C}_{2} \mathrm{H}_{2}\right)$ profiles obtained from neighboring lightcurves (e.g., the channel encompassing the second 3R-X Rydberg peak at $147.8 \mathrm{~nm})$. It seems certain that $f\left(\mathrm{C}_{2} \mathrm{H}_{2}\right)$ is falling off with decreasing pressure at the altitudes probed by the occultation.

Figure 7 presents the results on equatorial ethane mixing ratios. The uncertainties are too large and the pressure range of these results too narrow to enable us to say anything definite regarding the behavior of $f\left(\mathrm{C}_{2} \mathrm{H}_{6}\right)$ with height. The uncertainties are based both on variations among individual channels and variations caused by changing the channel offset valuc. There is a possibil- 
TABLE I

Empirical Equatorial Stratosphere Model

\begin{tabular}{|c|c|c|c|c|}
\hline $\begin{array}{c}\text { Altitude } \\
\text { (above } 1.0 \mathrm{bar}, \mathrm{km} \text { ) }\end{array}$ & $\begin{array}{c}n_{\mathrm{TOT}} \\
\left(10^{16} \mathrm{~cm}^{-3}\right)\end{array}$ & $\begin{array}{l}\text { Pressure } \\
\text { (mbar) }\end{array}$ & $\begin{array}{l}\text { Temperature } \\
\text { (K) }\end{array}$ & \\
\hline 366.1 & 0.19 & 0.035 & 130 & \\
\hline 358.8 & 0.23 & 0.039 & 126 & \\
\hline 351.5 & 0.27 & 0.045 & 124 & \\
\hline 344.2 & 0.31 & 0.052 & 122 & \\
\hline 336.9 & 0.36 & 0.060 & 120 & \\
\hline 329.6 & 0.42 & 0.069 & 120 & \\
\hline 322.3 & 0.48 & 0.080 & 119 & \\
\hline 315.0 & 0.56 & 0.092 & 120 & \\
\hline 307.7 & 0.64 & 0.106 & 120 & \\
\hline 300.4 & 0.73 & 0.122 & 121 & \\
\hline 293.2 & 0.83 & 0.140 & 122 & \\
\hline 285.9 & 0.95 & 0.161 & 123 & \\
\hline 278.6 & 1.07 & 0.185 & 125 & UVS \\
\hline 271.3 & 1.22 & 0.212 & 126 & \\
\hline 264.0 & 1.37 & 0.243 & 128 & \\
\hline 256.7 & 1.55 & 0.277 & 129 & \\
\hline 249.5 & 1.75 & 0.316 & 131 & \\
\hline 242.2 & 1.97 & 0.360 & 132 & \\
\hline 234.9 & 2.23 & 0.409 & 133 & \\
\hline 227.6 & 2.52 & 0.465 & 134 & \\
\hline 220.3 & 2.86 & 0.529 & 134 & \\
\hline 213.0 & 3.28 & 0.602 & 133 & \\
\hline 205.8 & 3.81 & 0.685 & 130 & \\
\hline 198.5 & 4.66 & 0.786 & 122 & \\
\hline 195.2 & 5.12 & 0.838 & 118 & \\
\hline 192.0 & 5.64 & 0.895 & 115 & \\
\hline 188.7 & 6.24 & 0.958 & 111 & \\
\hline 185.4 & 6.94 & 1.028 & 107 & \\
\hline 182.2 & 7.77 & 1.106 & 103 & Interpolated \\
\hline 178.9 & 8.75 & 1.194 & 99 & \\
\hline 175.7 & 9.90 & 1.293 & 95 & \\
\hline 172.4 & 11.3 & 1.405 & 90 & \\
\hline 169.1 & 12.8 & 1.533 & 87 & \\
\hline 165.9 & 14.6 & 1.679 & 83 & \\
\hline 162.6 & 16.7 & 1.846 & 80 & $\mathrm{RSS}^{a}$ \\
\hline
\end{tabular}

${ }^{a}$ Refer to Lindal et al. (1987, Table 1) for the density profile at lower altitudes.

ity that a fraction of the opacity leading to these mixing ratio values is actually due to $\mathrm{CH}_{4}$. Given the known photoabsorption cross sections, a methane mixing ratio in the range $10^{-7}$ to $3 \times 10^{-7}$ near 0.10 mbar could reduce the estimated ethane mixing ratios in the figure by up to a factor of 2 . However, there is not a systematic variation with decreasing wavelength among the residual opacities from the individual channels in the
137- to $142-\mathrm{nm}$ wavelength interval such as would be expected if methane were present in amounts greater than $10^{-7}$. Hence we conclude that ethane is responsible for the observed opacity.

\section{PHOTOCHEMICAL MODELS AND THE STRENGTH OF EDDY MIXING}

Photochemical calculations (1-D) have been carried out to infer the strength of eddy 


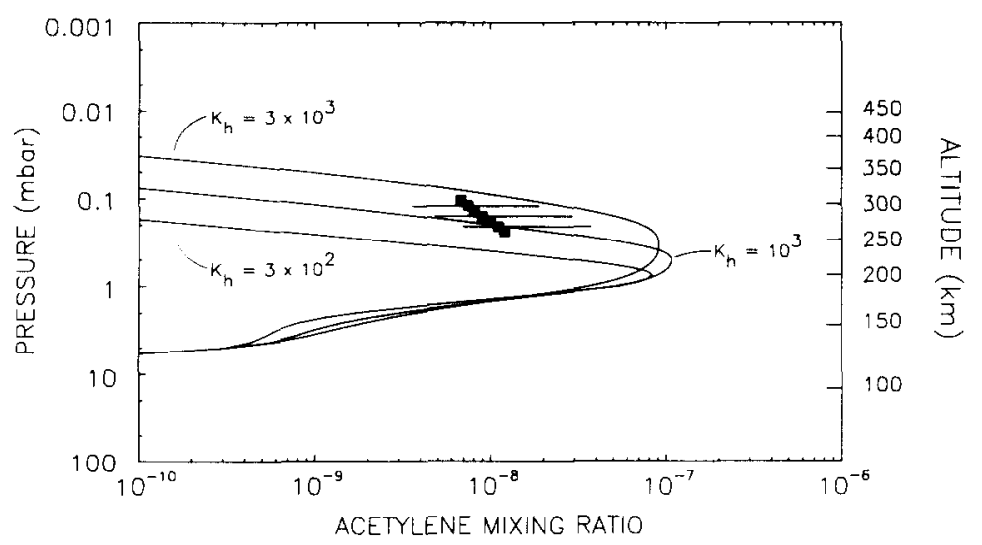

FIG. 6. Equatorial $\mathrm{C}_{2} \mathrm{H}_{2}$ mixing ratios. The data points are derived from the $\mathcal{N}_{\text {Los }}\left(\mathrm{C}_{2} \mathrm{H}_{2}\right)$ profile labeled " $p$ " in Fig. 3 in conjunction with the model stratosphere of Table 1 , at those altitudes where the opacity attributed to acetylene exceeds 0.10 with the total optical depth remaining less than 2.0 . The uncertainty estimates are described in the text. Pressure uncertainties are roughly indicated by symbol size. Photochemical model results for equatorial conditions at the time of the Uranian encounter are also shown, with $\beta=1 / 2$ in Eq. (3); the model curves are labeled by the assumed value of the eddy mixing coefficient at the methane homopause. Model inputs are described in the text.

mixing implied by the acetylene and ethane mixing ratios shown in Figs. 6 and 7, using the model stratosphere of Table I. The numerical photochemical code employed is a revised version of the scheme used in Herbert et al. (1987) and includes diacetylene as a reactant. It has recently been described (Atreya et al. 1990, Romani and Atreya 1989), so we will not go into details here. In brief, it solves the coupled continuity equations for methane and its photolysis products using an iterative Newton-Raphson

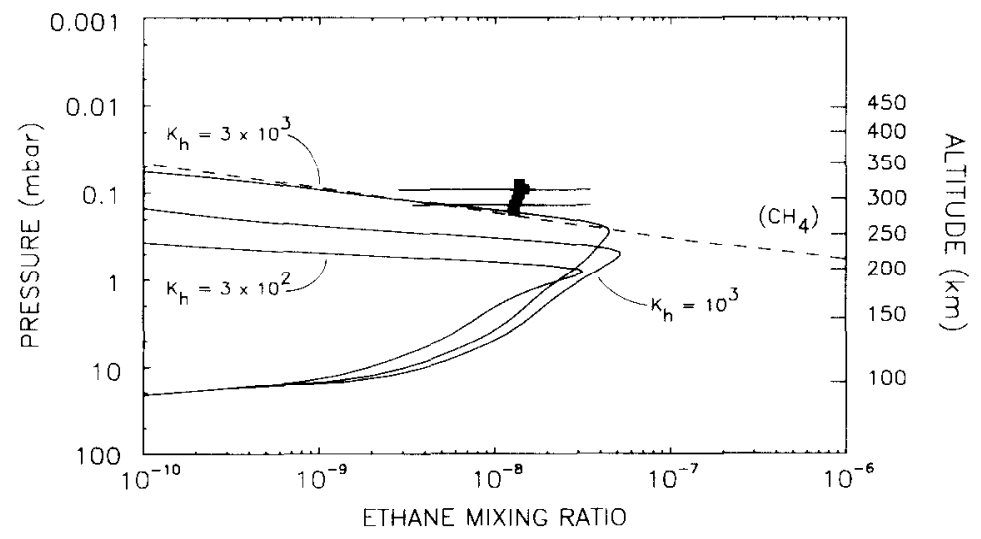

FIG. 7. Equatorial $\mathrm{C}_{2} \mathrm{H}_{6}$ mixing ratios. The data points are derived from the $\mathfrak{N}_{\mathrm{LOS}}\left(\mathrm{C}_{2} \mathrm{H}_{6}\right)$ profile of Fig. 3 in conjunction with the model stratosphere of Table I, at those altitudes where the opacity attributed to ethane exceeds 0.10 (methane may be contributing some absorption) with the total optical depth remaining less than 2.0. Acetylene opacity remains $\leq 0.10$. The uncertainty estimates are described in the text. The $f\left(\mathrm{C}_{2} \mathrm{H}_{6}\right)$ results for the photochemical calculations of Fig. 6 are also shown, along with the model $\mathrm{CH}_{4}$ mixing ratio profile for $K_{\mathrm{h}}=10^{3}$ (dashed curve); for $\mathrm{CH}_{4}$ to provide a significant fraction of the opacity attributed to $\mathrm{C}_{2} \mathrm{H}_{6}, f\left(\mathrm{CH}_{4}\right)$ must exceed $10^{-7}$ at the pressure levels of the UVS occultation data while the photochemical results suggest $f\left(\mathrm{CH}_{4}\right) \sim 10^{-8}$ 
TABLE II

Methane Equatorial Homopause Altitudes

\begin{tabular}{crc}
\hline $\begin{array}{c}K_{\mathrm{h}} \\
\left(\mathrm{cm}^{2} \mathrm{sec}^{-1}\right)\end{array}$ & $\begin{array}{c}z_{\mathrm{h}}^{a} \\
(\mathrm{~km})\end{array}$ & $\begin{array}{c}n_{\mathrm{TOT}} \\
\left(10^{16} \mathrm{~cm}^{-3}\right)\end{array}$ \\
\hline $10^{2}$ & 182 & 7.7 \\
$10^{3}$ & 289 & 0.89 \\
$10^{4}$ & 393 & 0.10 \\
$10^{5}$ & $\sim 512$ & $\sim 0.02$ \\
\hline
\end{tabular}

${ }^{a}$ Above 1.0-bar level.

procedure. Since it treats only vertical structure, its results should be interpreted in terms of global averages. Vertical transport includes both eddy mixing and molecular diffusion, with the former parameterized in terms of the eddy mixing coefficient. On Uranus, methane is transported into the stratosphere where solar ultraviolet photons (primarily Lyman- $\alpha$ ) convert it to the stable disequilibrium hydrocarbons $\mathrm{C}_{2} \mathrm{H}_{2}, \mathrm{C}_{2} \mathrm{H}_{6}$, $\mathrm{C}_{4} \mathrm{H}_{2}$, and higher order polyacetylenes. The lower stratosphere on Uranus is so cold that these species condense out to their respective ices. Condensation loss is included in the code for these species, which balances out the loss of methane via photolysis at the higher levels; however, condensation occurs at pressure levels far below those probed by occultation, so this process does not directly impinge upon the current comparisons with data. Summers and Strobel (1989) also discuss methane photochemistry under Uranian conditions and its implications for understanding UVS measurements; their photochemical scheme is generally similar to the one used here.

The variation of the eddy mixing coefficient $K(z)$ with height has been modeled with the relation

$$
K(z)=K_{\mathrm{h}} \times\left(\frac{n_{\mathrm{TOT}}\left(z_{\mathrm{h}}\right)}{n_{\mathrm{TOT}}(z)}\right)^{\beta},
$$

where the subscript $h$ refers to the methane homopause level. Some representative methane homopause altitudes $z_{\mathrm{h}}$ for the model stratosphere of Table I are given in Table II. The photochemical mixing ratio profiles shown in Figs. 6 and 7 were obtained with $\beta=1 / 2$. Evidently, the $K_{\mathrm{h}}=10^{3} \mathrm{~cm}^{2}$ $\sec ^{-1}$ case gives fair agreement with the acetylene data but the ethane mixing ratios in these models are too low. This turns out to be a persistent difficulty in our attempts to fit photochemical distributions to the data, a difficulty also exhibited by the photochemical models of Summers and Strobel (1989); further examples are given below. In these calculations, the mixing ratio of methane at the tropopause was set to $3 \times 10^{-5}$, in accord with the $\sim 30 \%$ saturation level deduced by Lindal et al. (1987) at the same latitude. A solar zenith angle of $83^{\circ}$ was used in evaluating the slant factor for incident solar radiation, and diurnal averaging was applied; the incident solar Lyman- $\alpha$ flux was taken to be $2.7 \times 10^{11}$ photons $\mathrm{cm}^{-2}$ $\mathrm{sec}^{-1}$ at 1.0 AU, corresponding to the solar minimum period of the encounter. The downward flux of atomic hydrogen from the thermosphere was set to $3 \times 10^{7} \mathrm{~cm}^{-2} \mathrm{sec}^{-1}$ (Herbert et al. 1987); for all other species, the flux at the upper boundary of the photochemical zone was set to zero. We note that the $\mathrm{C}_{2} \mathrm{H}_{2}$ and $\mathrm{C}_{2} \mathrm{H}_{6}$ mixing ratio profiles generated by the photochemical code are relatively stable with regard to variations in these quantities. For example, reducing the tropopause methane mixing ratio from $3 \times$ $10^{-5}$ to $10^{-5}$, which would be more in keeping with the vapor pressure of methane near the tropopause temperature minimum of 49 $\mathrm{K}$ near $30^{\circ} \mathrm{S}$ latitude (Flasar et al. 1987), alters model acetylene and ethane mixing ratios at the pressure levels of the UVS occultation data by less than a factor of two.

By varying $K_{\mathrm{h}}$ with $\beta$ prescribed, keeping other parameters fixed, mixing ratio profiles have been found that pass near the center of the $\mathrm{C}_{2} \mathrm{H}_{2}$ data points; these model results are shown in Figs. 8 and 9. Constant eddy mixing profiles $(\beta=0)$ have been considered by Summers and Strobel (1989), while the $\beta>0$ cases all have $K<200 \mathrm{~cm}^{2} \mathrm{sec}^{-1}$ near the 3-mbar level. It is evident in Fig. 8 that none of the model profiles reproduce the variation of mixing ratio with pressure 


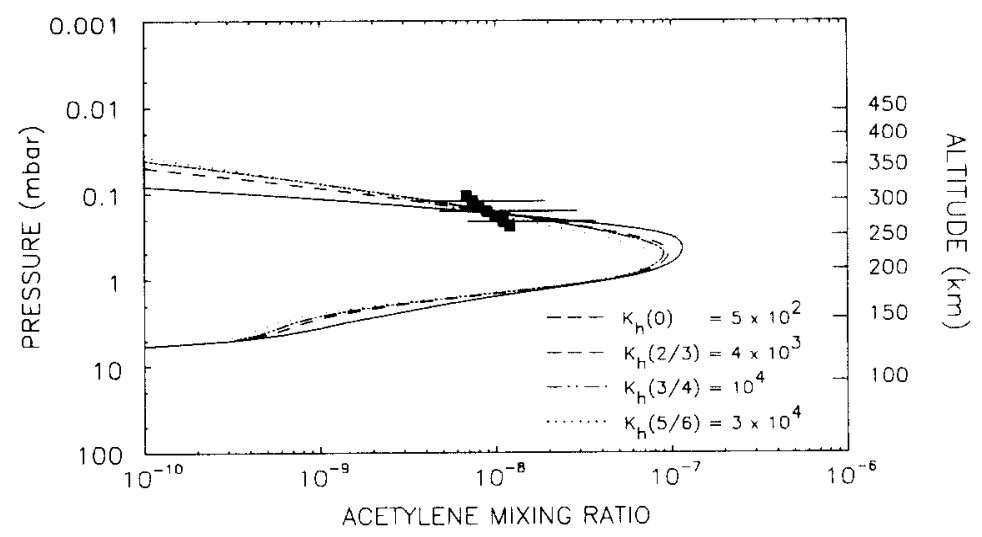

FIG. 8. Further photochemical models of the equatorial $\mathrm{C}_{2} \mathrm{H}_{2}$ distribution. In these calculations, the $K_{\mathrm{h}}$ value was varied with $\beta$ in $\mathrm{Eq}$. (3) held fixed until a mixing ratio profile passing near the center of the acetylene data points was obtained (for $\beta=1 / 2$, see Fig. 6). The labeling convention in this figure is $K_{\mathrm{h}}(\beta)$.

suggested by the $\mathrm{C}_{2} \mathrm{H}_{2}$ data. Further, the model $\mathrm{C}_{2} \mathrm{H}_{6}$ mixing ratios are consistently too low (Fig. 9). We have also carried out photochemical calculations with tropopause methane mixing ratios well below saturation, as has been suggested to be the case in the dayside polar region (Yelle et al. 1989). Representative results are shown in Fig. 10, for $f\left(\mathrm{CH}_{4}\right)=10^{-6}$ at the tropopause and $\beta=1 / 2$. The best fit to the acetylene data is obtained with $K_{\mathrm{h}}=8 \times 10^{3} \mathrm{~cm}^{2} \mathrm{sec}^{-1}$; however, there is clearly not enough ethane generated in this model to account for the observed opacity at wavelengths 137-142 nm. Reducing $f\left(\mathrm{CH}_{4}\right)$ further worsens the disagreement by lowering the level for a photochemical optical depth of unity, thereby requiring stronger eddy mixing to bring the hydrocarbons up to the pressure levels of the UVS occultation data.

In comparing Figs. 6-10, the following points can immediately be drawn: First, while it is not possible to say anything regarding the strength of eddy mixing at levels

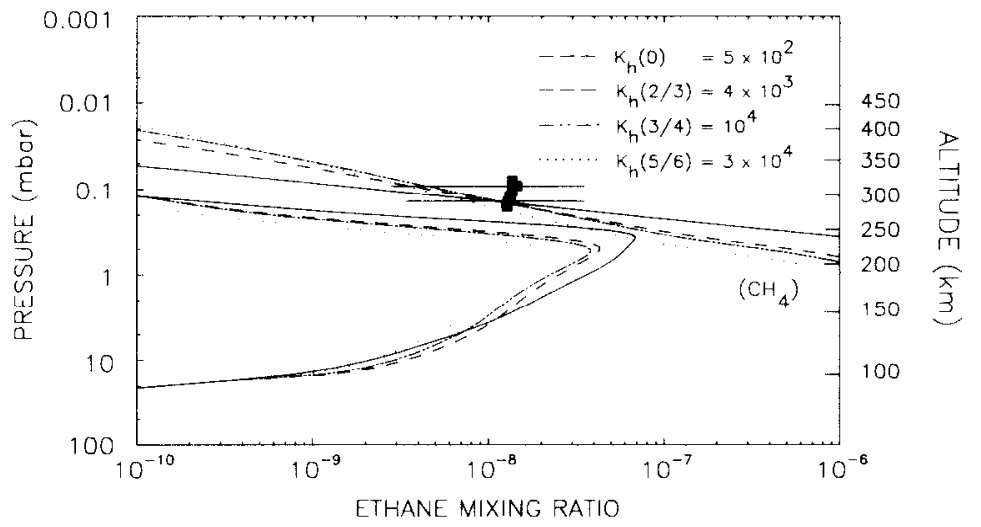

FIG. 9. The $f\left(\mathrm{C}_{2} \mathrm{H}_{6}\right)$ and $f\left(\mathrm{CH}_{4}\right)$ results for the photochemical calculations of Fig. 8 . The labeling convention is $K_{\mathrm{h}}(\beta)$. 


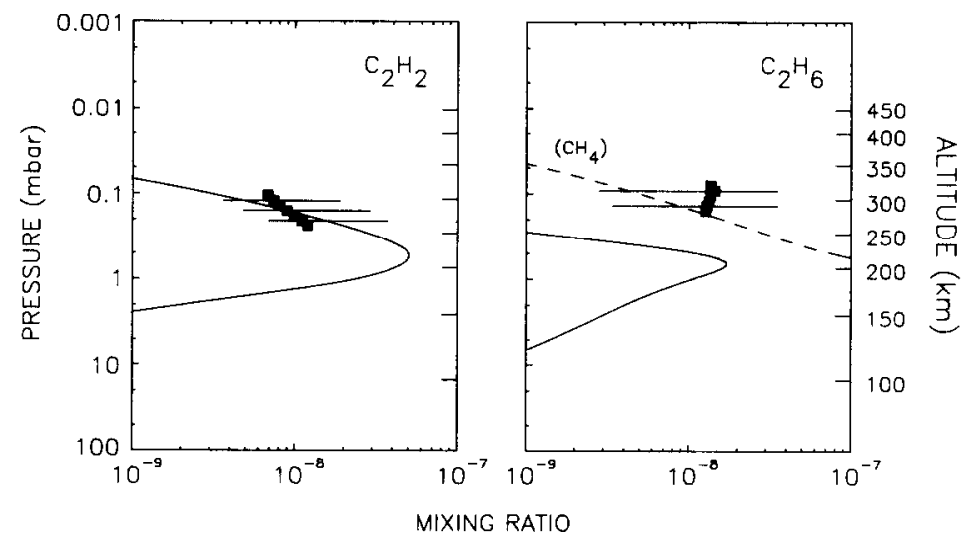

FIG. 10. Photochemical model rcsults $\left(\beta=1 / 2, K_{\mathrm{h}}=8 \times 10^{2} \mathrm{~cm}^{2} \mathrm{sec}^{-1}\right.$ giving the best agreement with the acetylene data) for $f\left(\mathrm{C}_{2} \mathrm{H}_{2}\right), f\left(\mathrm{C}_{2} \mathrm{H}_{6}\right)$, and $f\left(\mathrm{CH}_{4}\right)$ (dashed curve) for $\sim 1 \% \mathrm{CH}_{4}$ relative humidity at the tropopause. While such a low value of the tropopause methane mixing ratio is not inconsistent with the acetylene data) for $f\left(\mathrm{C}_{2} \mathrm{H}_{2}\right), f\left(\mathrm{C}_{2} \mathrm{H}_{6}\right)$. and $f\left(\mathrm{CH}_{4}\right)$ (dashed curve) for $\sim 1 \% \mathrm{CH}_{4}$ relative humidity for the observed $\mathrm{C}_{2} \mathrm{H}_{6}$ opacity.

closer to the tropopause, the acetylene results suggest that the value of the eddy mixing coefficient at the methane homopause within the context of our 1-D photochemical scheme is somewhere between $10^{3} \mathrm{~cm}^{2}$ $\mathrm{sec}^{-1}$ and $10^{4} \mathrm{~cm}^{2} \mathrm{sec}^{-1}$. Summers and Strobel (1989) argued for $K_{\mathrm{h}}=3 \times 10^{3} \mathrm{~cm}^{2} \mathrm{sec}^{-1}$ $(\beta=1 / 2)$ to address simultaneously the $\mathrm{C}_{2} \mathrm{H}_{2}$ and $\mathrm{CH}_{4}$ (upper limit) mixing ratio estimates of Herbert et al. (1987) for global mean illumination conditions. Second, the long timescale for vertical transport near the 0.10 -mbar level implied by $K_{\mathrm{h}} \approx 10^{3} \mathrm{~cm}^{2}$ $\sec ^{-1}\left(\sim 2 \times 10^{10} \mathrm{sec}\right)$ in turn implies that the distribution of hydrocarbons in the upper stratosphere is largely governed by photochemistry, which is characterized by the timescales for chemical loss and production of methane $\left(-2 \times 10^{8} \mathrm{sec}\right)$ (see also Summers and Strobel (1989)). Photochemical timescales are also short compared with the timescale for changing illumination conditions (i.e., the Uranian year, $2.6 \times 10^{9} \mathrm{sec}$, given the high obliquity of this planet), which has guided our selection of conditions applied in the photochemical calculations. We note in comparison that the Summers and Strobel (1989) model for near-terminator equatorial conditions (model $\mathrm{F}\left(\chi=80^{\circ}\right.$ ) with $K_{\mathrm{h}}=1500 \mathrm{~cm}^{2} \mathrm{sec}^{-1}$ and $\beta=1 / 2$ ) fails to provide the observed mixing ratio of acetylene near 0.10 mbar by at least an order of magnitude; evidently, there are important differences between the photochemical codes beyond the handling of condensation, the effects of which alone cannot account for the differences in model $\mathrm{C}_{2} \mathrm{H}_{2}$ mixing ratios because of the separation in pressure between the occultation and condensation levels and the disparity between mixing vs photochemical timescales. Third, the photochemical models suggest $f\left(\mathrm{CH}_{4}\right)<10^{-7}$ at the pressure levels of the UVS occultation data. The model methane mixing ratios shown in Figs. 7, 9, and 10 are all in the neighborhood of $10^{-8}$, which is too small to result in observable extinction at wavelengths $137-142 \mathrm{~nm}$, in accord with the lack of a systematic increase in residual opacity with decreasing wavelength in the corresponding UVS lightcurves noted earlier. These conclusions are subject to a caveat regarding transport by a meridional circulation system like the one suggested by Flasar et al. (1987); this is discussed below. In any event, ethane has definitely been detected, and the strength of eddy mixing implied by the observed acetylene abundance fails to 
account for the observed ethane abundance at least when viewed from the perspective of one-dimensional photochemical models.

\section{SUMMARY AND DISCUSSION}

On Uranus, the low abundance of stratospheric hydrocarbons leaves $\mathrm{H}_{2}$ Rayleigh scattering as a major source of FUV opacity at stratospheric pressures. This paucity is due to weak vertical mixing and cold tropopause temperatures, and is such that analysis of $\mathrm{H}_{2}$ Rayleigh scattering opacity profiles can provide the vertical structure of the $\mathrm{H}_{2}-\mathrm{He}$ background atmosphere in the photochemical region. We have reanalyzed the solar occultation lightcurves obtained with the UVS instrument during the Voyager encounter to take advantage of this coincidence. Yelle et al. (1989) also exploited this coincidence in their analysis of an FUV emission-reflection spectrum of high $S / N$ obtained from the subsolar (polar) region with the same instrument. The occultation results pertain to the equatorial upper stratosphere (0.03-0.60 mbar) while albedo measurements probe deeper levels below 1.0 mbar (Caldwell et al. 1988, Yelle et al. 1989). The results of the our analysis are summarized in Table $I$ and Figs. 6-10. In Table I, total number densities, pressures, and atmospheric temperatures derived from the UVS $\mathrm{H}_{2}$ Rayleigh scattering lightcurves are given, along with an interpolation to the RSS equatorial atmosphere model at altitudes below $160 \mathrm{~km}$ (Lindal et al. 1987). This model stratosphere has been used in photochemical calculations to infer the strength of eddy mixing near the top of the photochemical region implied by acetylene $\left(\mathrm{C}_{2} \mathrm{H}_{2}\right)$ and ethane $\left(\mathrm{C}_{2} \mathrm{I}_{6}\right)$ mixing ratios also derived from UVS occultation lightcurves; these are displayed in Figs. 6-10, along with mixing ratio profiles generated by the photochemical code for various assumptions regarding the strength of eddy mixing. The main advance over the Herbert et al. (1987) analysis of these measurements is in specifying the pressure range wherein hydrocar- bon signatures are clearly present in the data, and in refining estimates of the uncertainties associated with the derived mixing ratios.

Prior to the Voyager 2 encounter with Uranus, estimates of hydrocarbon abundances in the Uranian upper atmosphere had been derived from Earth-based measurements of the UV albedo and the IR brightness spectrum. Several sets of measurements of the Uranian infrared emission spectrum (7-14 $\mu \mathrm{m}$ ) have recently been collected (Orton et al. 1990), with the aim of settling questions regarding absolute calibration raised by Orton et al. (1987). These measurements were also compared with calculated brightness spectra obtained with stratospheric models wherein hydrocarbons were uniformly mixed (depleted where required by local saturation equilibrium) and with models incorporating photochemical distributions of hydrocarbons (computed using the scheme described in Atreya et al. (1990)). The IR data taken together suggest $f\left(\mathrm{C}_{2} \mathrm{H}_{2}\right) \approx 1.5$ $\times 10^{8}$ with an uncertainty factor of 3.4 and $f\left(\mathrm{C}_{2} \mathrm{H}_{6}\right) \leq 10^{-8}$, when interpreted using the uniformly mixed models. Analysis of the UV albedo (170-200 nm) derived from IUE measurements by Caldwell et al. (1988) suggested acetylene mixing ratios of $1 \times 10^{-8}$ with an uncertainty of roughly a factor of two, again assuming $\mathrm{C}_{2} \mathrm{H}_{2}$ to be uniformly mixed in the stratosphere. It is important to remember when comparing these estimates with our results that Earth-based observations provide global averages of "mean" stratospheric mixing ratios; photochemical distributions for acetylene, for instance, could exhibit peak mixing ratios well in excess of "mean" values and remain consistent with the data. In fact, the IR brightness spectra comparisons in Orton et al. (1990) used two distinct photochemical hydrocarbon distributions, one based on the Herbert et al. (1987) analysis of the UVS solar occultation data and the other geared toward meeting constraints derived from the UVS polar reflection spectrum analysis of Yelle $e t$ al. (1989). Both models gave $\mathrm{C}_{2} \mathrm{H}_{2}$ emissions 
near $13.7 \mu \mathrm{m}$ too dim to match the data, with the "polar" model being particularly deficient. This implies that peak acetylene mixing ratios of $10^{-7}$ could be easily accomodated, subject to uncertainties regarding the temperature structure. In brief, our equatorial results are not inconsistent with the Earth-based results and are likely to be more representative of the global average than the polar results of Yelle et al. (1989).

The inability of our photochemical models to account for the observed ethane mixing ratios near 0.10 mbar is most likely due to the simple picture of vertical transport built into the photochemical code. This points to the necessity of invoking an additional transport mechanism; a stratospheric circulation system would seem to be the most likely candidate. Flasar et al. (1987) have oullined a meridional circulation system compatible with Voyager 2 IRIS measurements, although it is not clear if that system would extend to the pressure levels of the occultation data. Ethane tends to be more stable than acetylene under Uranian stratospheric conditions, in the sense that timescales for photochemical $\mathrm{C}_{2} \mathrm{H}_{6}$ production and destruction are longer than for $\mathrm{C}_{2} \mathrm{H}_{2}$. Thus, ethane is more likely to show departures from a photochemical distribution in the presence of a stratospheric circulation system. Yelle et al. (1989) also discuss the need to invoke the existence of a stratospheric circulation system to account for the distribution of hydrocarbons in the Uranian stratosphere. While probing deeper levels, their analysis of the FUV polar reflection spectrum suggests "mean" $\mathrm{C}_{2} \mathrm{H}_{2}$ mixing ratios consistent with our results; however, as previously noted, their analysis also requires the methane mixing ratio at the tropopause to be at least an order of magnitude lower than the amount needed to provide the observed abundance of ethane near 0.10 mbar at the equator. To account for the apparent strong latitudinal gradient in stratospheric methane abundance, Yelle et al. seized upon the meridional circulation system of Flasar et al. (1987) and proposed that methane was injected into the stratosphere mainly at the latitude of the tropopause temperature minimum $\left(25^{\circ} \mathrm{S}\right)$ with subsequent photochemical depletion in transit to the polar zone (transport time $\sim 10^{11} \mathrm{sec}$ ). While it is beyond the purpose of this paper to comment on the particular scheme proposed by Yelle et al.(1989), it is obvious that a more comprehensive 2-D photochemicaltransport study is required to fully understand the UVS Uranian observations. Matching the ethane mixing ratios from the UVS solar occultation analysis presented in this paper will be a stringent test for any such study.

\section{$\Lambda$ CKNOWLEDGMENTS}

We thank L. Trafton, for catching an error in an earlier version. This research has been supported by NASA Grants NAGW-1504 and NAGW-1771 (J.B. and S.K.A), NAGW-1208 (F.H.), and NASA Contract NAS5-30134 (P.R.).

\section{REFERENCES}

AtreyA, S. K. 1986. Atmospheres and Ionospheres of the Outer Planets and their Satellites. SpringerVerlag, Berlin.

Atreya, S. K., T. M. Donahue, and M. C. Festou 1981. Jupiter: Structure and composition of the upper atmosphere. Astrophys. J. 247, L43-L47.

Atreya, S. K., B. R. Sandel, and P. N. Romani 1990. Photochemistry and vertical mixing. In Urants (J. T. Bergstralh and M. S. Matthews, Eds.) Univ. of Arizona, Tucson.

Baron, R. L., and R. G. Frfnch 1989. New Uranian atmospheric temperatures from Earth-based stellar occultations using Voyager 2 EUV initial conditions. Bull. Amer. Astro. Soc. 21, 916.

Broadfoot, A. L., B. R. Sandel, D. E. Shemansky, S. K. Atreya, T. M. Donahue, H. W. Moos, J. L. Bertaux, J. E. Blamont, J. M. Ajello, D. F. Strobel, J. C. McConnell, A. Dalgarno, R. Goody, M. B. McElroy, AND Y. L. YUNG 1977. Ultraviolet spectrometer experiment for the Voyager mission. Space Sci. Rev. 21, 183-205.

Broadfoot, A. L., B. R. Sandel, D. E. Shemansky, J. C. McConnell, G. R. Smith, J. B. HolberG, S. K. Atreya, T. M. Donahue, D. F. Strobel, AND J. L. BERTAUX 1981. Overview of the Voyager ultraviolet spectrometry results through Jupiter encounter. J. Geophys, Res. 86, 8259-8284.

Broadfoot, A. L., F. Herbert, J. B. HolberG, D. M. Hunten, S. Kumar, B. R. Sandel, D. E. Shemansky, G. R. Smith, R. V. Yelle, D. F. 
Strobel, H. W. Moos, T. M. Donahue, S. K. Atreya, J. L. Bertaux, J. E. Blamont, J. C. MCConnell, A. J. Dessler, S. Linick, and R. Springer 1986. Ultraviolet spectrometer observations of Uranus. Science 233, 74-79.

Caldwell, J., R. Wagener, and K. H. Fricke 1988. Observations of Nepture and Uranus below $2000 \AA$ with the IUE. Icarus 74, 133-140.

Elliot, J. L., I. S. Glass, R. G. French, and J. A. KANGAS 1987. The occultation of KME 17 by Uranus and its rings. Icarus 71, 91-102.

Festou, M. C., ANd S. K. Atreya 1982. Voyager ultraviolet stellar occultation measurements of the composition and thermal profiles of the Saturnian upper atmosphere. Geophys. Res. Lett. 9, 1147-1150.

Flasar, F. M., B. J. Conrath, P. J. Gierasch. and J. A. Pirraglia 1987. Voyager infrared observations of Uranus' atmosphere: Thermal structure and dynamics. J. Geophys. Res. 92, 15,011-15,018.

Ford, A. L., AND J. C. Browne 1973. Rayleigh and Raman cross sections for the hydrogen molecule. Atomic Data 5, 305-313.

French, R. G., J. L. Elliot, E. W. Dunham, D. A. Allen, J. H. Elias, J. A. Frogel, and W. Liller 1983. The thermal structure and energy balance of the Uranian upper atmosphere. Icarus 53, $399 \ldots 414$.

French, R. G., T. J. Jones, AND A. R. Hyland 1987. The 1 May 1982 stellar occultation by Uranus and the rings: Observations from Mount Stromlo observatory. Icarus 69, 499-505.

hanel, R., B. Conrath, F. M. Flasar, V. Kunde, W. Maguire, J. Pearl, J. Pirraglia, R. Samuelson, D. Cruikshank, D. Gautier, P. Gierasch, L. HoRn, AND P. SChUlte 1986. Infrared observations of the Uranian system. Science 233, 70-74.

Hays, P. B., AND R. G. Roble 1968. Atmospheric properties from the inversion of planetary occultation data. Planet. Space Sci. 16, 1197-1198.

herbert, F., B. R. Sandel, R. V. Yeli.e, J. B. HolBERG, A. L. Broadfoot, D. E. Shemansky, S. K. Atreya, and P. N. Romani 1987. The upperatmosphere of Uranus: EUV occultations observed by Voyager 2. J. Geophys. Res. 92, 15,093-1.5,109.
Heroux, L., and H. E. Hinteregger 1978. Acronomical reference spectrum for solar UV below 2000 angstroms. J. Geophys. Res. 83, 5305-5308.

Lindal, G. F., J. R. Lyons, D. N. SwEETnam, V. R. Eshleman, D. P. Hinson, and G. L. Tyler 1987. The atmosphere of Uranus: Results of radio occultation measurements with Voyager 2. J. Geophys. Res. 92, 14,987-15,001.

Millis, R. L., L. H. Wasserman, and R. G. FrenCH 1987. Observations of the 22 April 1982 stellar occultation by Uranus and the rings. Icarus 69, 176-184.

Orton, G. S., D. K. Aitken, C. Smith, P. F. Roche, J. CALDWELL, AND R. SNYDER 1987. The spectra of Uranus and Neptune at $8-14$ and $17-23 \mu \mathrm{m}$. Icarus 70, $1-12$.

Orton, G. S., K. H. Baines, J. Caldwell, P. Romani, A. T. Tokunaga, and R. A. West 1990. Calibration of the 7- to $14-\mu \mathrm{m}$ brightness spectra of Uranus and Neptune. Icarus 85, 257-265.

Pollack, J. B., K. Rages, S. K. Pope, M. G. Tomasko, P. N. Romani, and S. K. Atreya 1987. Nature of the stratospheric haze on Uranus: Evidence for condensed hydrocarbons. J. Geophys. Res. 92, 15,037-15,065.

Press, W. H., B. P. Flannery, S. A. Teukolsky, AND W. H. VetTERLING 1986. Numerical Recipes: The Art of Scientific Computing. Cambridge Univ. Press, Cambridge.

Romani, P. N., AND S. K. Atreya 1989. Stratospheric aerosols from $\mathrm{CH}_{4}$ photochemistry on Neptune. Geophys. Res. Lett. 16, 941-944.

Sicardy, B., M. Combes, J. Lecacheux, P. Bouchet, A. Brahic, P. Laques, C. Perrier, L. VAPILLON, AND Y. ZEAU 1985. Variations of the stratospheric temperature along the limb of Uranus: Results of the 22 April 1982 stellar occultation. Icarus 64, 88-106.

Summers, M. E., and D. F. Strobel 1989. Photochemistry of the atmosphere of Uranus. Astrophys. J. 346, 495-508.

Yelle, R. V., J. C. McConnell, D. F. Strobel, AND L. R. Doose 1989. The far ultraviolet reflection spectrum of Uranus: Results from the Voyager encounter. Icarus 77, 439-456. 